\title{
Diffuse interstellar bands in M 33
}

\author{
M. A. Cordiner ${ }^{1}$, K. T. Smith ${ }^{2}$, N. L. J. Cox ${ }^{3}$, C. J. Evans ${ }^{4}$, I. Hunter ${ }^{1}$, N. Przybilla ${ }^{5}$, F. Bresolin ${ }^{6}$, and P. J. Sarre ${ }^{2}$ \\ 1 Astrophysics Research Centre, School of Mathematics and Physics, Queen's University, Belfast, BT7 1NN, UK \\ e-mail: m.cordiner@qub.ac.uk \\ 2 School of Chemistry, The University of Nottingham, University Park, Nottingham, NG7 2RD, UK \\ 3 Herschel Science Centre, European Space Astronomy Centre, ESA, PO Box 78, 28691 Villanueva de la Cañada, Madrid, Spain \\ ${ }^{4}$ UK ATC, Royal Observatory Edinburgh, Blackford Hill, Edinburgh, EH9 3HJ, UK \\ 5 Dr. Remeis-Sternwarte Bamberg, Sternwartstr. 7, 96049 Bamberg, Germany \\ 6 Institute for Astronomy of the University of Hawaii, 2680 Woodlawn Drive, 96822 Honolulu, HI, USA \\ Received 3 September 2008 / Accepted 10 October 2008
}

\section{ABSTRACT}

\begin{abstract}
Aims. We investigate the diffuse interstellar band (DIB) spectrum in the interstellar medium of the Local Group spiral galaxy M 33. Methods. Optical spectra of the M33 supergiant star J013346.96+303642.8 were taken at a resolving power of $~ 3000$ using the DEIMOS spectrograph of the W. M. Keck Observatory.

Results. We report the first detection and measurement of DIBs in M 33. The $\lambda \lambda 5705,5780,5797,6203,6269,6283$, and 6613 DIBs are detected at velocities matching the M 33 Na I absorption lines. The 14428 DIB is tentatively detected. The stellar spectrum is classified as A3 Ia and the line-of-sight interstellar reddening is derived. Diffuse interstellar band (DIB) equivalent widths and radial velocities are presented.

Conclusions. The overall spectrum of DIBs observed in M33 is found to be similar to that observed in the Milky Way. However, along this line-of-sight, the M 33 DIB equivalent widths per unit reddening are large compared with those typically observed in the Galaxy.
\end{abstract}

Key words. astrochemistry - galaxies: Local Group - galaxies: ISM - ISM: lines and bands - ISM: atoms - ISM: dust, extinction

\section{Introduction}

More than 300 diffuse interstellar bands (DIBs) are now known but the carriers have remained unidentified since their discovery almost 90 years ago. It is debated whether the DIB carriers arise from dust, gas, or the large-molecule component of the interstellar medium (see the review by Sarre 2006). The substructure present in many of the DIB profiles indicates that they are caused by large gas-phase molecules (Sarre et al. 1995; Ehrenfreund \& Foing 1996).

Extragalactic DIB research is useful because it broadens the range of physical and chemical conditions with respect to which the properties of the carriers may be analysed. Previous studies of DIBs outside the Milky Way (MW) were reviewed by Snow (2002). Recent research on the relationships between atoms, molecules, dust and DIBs in external galaxies has focused on the Large and Small Magellanic Clouds (e.g. Ehrenfreund et al. 2002; Cox et al. 2006, 2007; Welty et al. 2006). Beyond the Magellanic Clouds, studies are few, and have generally been confined to sightlines probed by bright supernovae (e.g. Sollerman et al. 2005; Cox \& Patat 2008), or background quasars (e.g. York et al. 2006). Based on data recorded in the same observing run as the present study, Cordiner et al. (2008) made the first measurements of diffuse interstellar bands in M 31 and measured DIB equivalent widths along two sightlines that were considerably stronger per unit $E_{B-V}$ than the MW average.

M 33 presents the opportunity to study the effects on the DIB carriers of the chemical and physical conditions found in this Local Group spiral galaxy. This Letter presents the spectrum of the supergiant star J013346.96+303642.8 in M 33 and we report the first detection of DIBs in the M33 galaxy. The observed DIB properties are discussed in relation to those in other galaxies and the physical and chemical conditions in the M 33 ISM.

\section{Observations}

Forty-three bright stars were observed throughout the disc of M 33 in November 2003 using the Keck DEIMOS spectrograph. Two angles of the $1200 \mathrm{G}$ grating were used to cover the wavelength regions of approximately 3500-6300 and 6000-9000 with a spectral resolution $\Delta \lambda \approx 1.8 \AA$ (corresponding to a resolving power between 2200 and 4500). The total exposure time was $2.25 \mathrm{~h}$ in the blue region and $1.5 \mathrm{~h}$ in the red, during which the seeing was $0.5-0.8^{\prime \prime}$. The data were reduced using the deep2 pipeline then Doppler-corrected to the LSR frame. Table 1 shows the co-ordinates and photometry of J013346.96+303642.8 (from Massey et al. 2006). This target was selected for analysis in this Letter because its spectrum shows the most prominent DIBs of the forty-three stars observed. The star is spatially well-resolved and distinct from any neighbouring objects that might have contaminated its spectrum or photometry. The rest of the data will be presented elsewhere.

\section{Analysis and results}

\subsection{Stellar spectral type}

The spectrum of J013346.96+303642.8 was classified with reference to the Galactic standards of Evans \& Howarth (2003), with luminosity class assigned on the basis of the $\mathrm{H} \gamma$ equivalent width (Evans et al. 2004). The stellar radial velocity of 
Table 1. Sightline parameters for J013346.96+303642.8.

\begin{tabular}{ccccccccc}
\hline \hline $\begin{array}{c}\text { RA } \\
\mathrm{J} 2000\end{array}$ & $\begin{array}{c}\text { Dec } \\
\mathrm{J} 2000\end{array}$ & $\begin{array}{c}V \\
(\mathrm{mag})\end{array}$ & $\begin{array}{c}B-V \\
(\mathrm{mag})\end{array}$ & Sp. Type & $\begin{array}{c}E_{B-V}^{\mathrm{Tot}} \\
(\mathrm{mag})\end{array}$ & $\begin{array}{c}E_{B-V}^{\mathrm{M} 33} \\
(\mathrm{mag})\end{array}$ & $\begin{array}{c}v_{*} \\
\left(\mathrm{~km} \mathrm{~s}^{-1}\right)\end{array}$ & $\begin{array}{c}S / N \\
@ 5800 \AA\end{array}$ \\
\hline $01: 33: 46.96$ & $+30: 36: 42.8$ & $17.965 \pm 0.004$ & $0.332 \pm 0.004$ & A3 Ia & $0.26 \pm 0.02$ & $0.22 \pm 0.04$ & $-134 \pm 1$ & 80 \\
\hline
\end{tabular}

Derived stellar spectral type and LSR radial velocity $\left(v_{*}\right)$ are given. $E_{B-V}^{\mathrm{Tot}}$ is the total line-of-sight reddening. $E_{B-V}^{\mathrm{M} 33}$ is the reddening associated with interstellar matter in M 33 (corrected for the Galactic foreground - see Sect. 3.4).

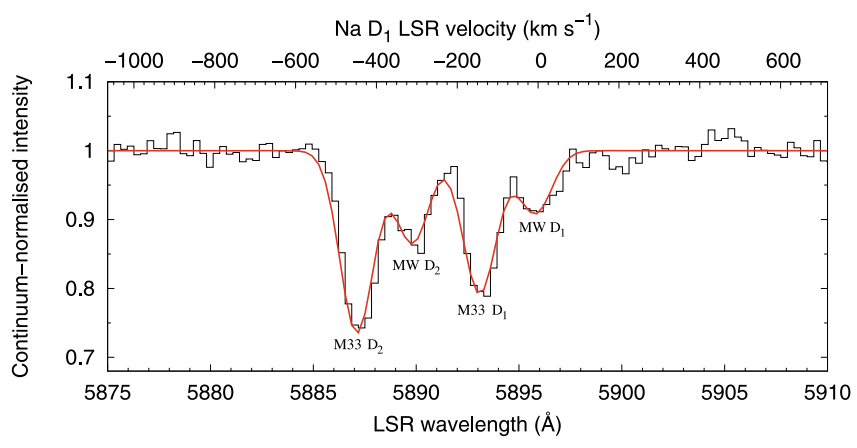

Fig. 1. Observed continuum-normalised $\mathrm{Na} \mathrm{D}$ spectrum of J013346.96+303642.8 (histogram) with best-fitting model overlaid (red curve). The velocity scale at the top is relative to the $\mathrm{Na} \mathrm{D}_{1}$ rest wavelength. The M33 and Galactic (MW) absorption components are labelled.

$-134 \mathrm{~km} \mathrm{~s}^{-1}$ is the mean of the H I Paschen lines, with a standard error of $1 \mathrm{~km} \mathrm{~s}^{-1}$.

We analysed Galactic stellar spectra of the same spectral type as the target for the presence of lines overlapping the detected DIBs. No significant contamination of the $\lambda \lambda 5705,6196$, $6203,6269,6283$ or 6613 DIBs is expected. The $\lambda 5780$ and $\lambda 5797$ DIBs may suffer contamination of up to about 5 and $2 \mathrm{~m} \AA$, respectively, as a result of overlapping lines of Fe I. Due to its large width, $\lambda 4428$ is overlapped by numerous strong stellar lines.

\subsection{Interstellar sodium $D$ lines}

The observed Na D lines (see Fig. 1) show absorption components due to interstellar gas at velocities consistent with the Galactic and M 33 ISM (around radial velocities of 0 and $-150 \mathrm{~km} \mathrm{~s}^{-1}$ respectively). The $\mathrm{Na} \mathrm{D}$ spectra were modelled using VAPID (Howarth et al. 2002), and the resulting fits are plotted. The mean $\mathrm{Na}$ I component radial velocities and the equivalent width $(E W)$ of the M33 $\mathrm{D}_{1}$ absorption component are given in Table 2. The interstellar Na D lines are contaminated by stellar photospheric Na I. From stellar spectral modelling using SYNSPEC and Kurucz (1993) model atmospheres with solar metallicity, we estimate the stellar contribution to the $\mathrm{Na} \mathrm{D}_{1}$ line $E W$ to be at least $160 \mathrm{~m} \AA$ (which corresponds to $\gtrsim 40 \%$ of the total $\mathrm{NaI}$.

\subsection{Diffuse interstellar bands}

We examined the spectrum of J013346.96+303642.8 for all strong DIBs in the survey of Jenniskens \& Desert (1994) (including all with central depths greater than 0.05 in their Fig. 6). Scaled to the target's reddening, this central depth limit corresponds to $\sim 1 \sigma$ of the spectral Poisson noise. The $\lambda \lambda 5705$, 5780, 5797, 6203, 6269, 6283 and 6613 DIBs were detected close to the M33 Na I velocity. Both $\lambda 6196$ and $\lambda 4428$ were
Table 2. Measured and literature $\mathrm{DIB}$ and $\mathrm{Na} \mathrm{D}_{1}$ line parameters.

\begin{tabular}{|c|c|c|c|c|}
\hline & \multicolumn{2}{|c|}{ M33 } & \multicolumn{2}{|c|}{ 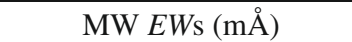 } \\
\hline & $v\left(\mathrm{~km} \mathrm{~s}^{-1}\right)$ & $E W(\mathrm{~m} \AA)$ & $\beta^{1} \mathrm{Sco}$ & MW Avg.@ $E_{B-V}^{\mathrm{M} 33}$ \\
\hline 4428 & $-144^{f}$ & $<1550$ & & 595 \\
\hline 5705 & $-143_{-15}^{+15}$ & $46_{-18}^{+18}$ & 23 & 26 \\
\hline 5780 & $-130_{-19}^{+15}$ & $282_{-38}^{+36}$ & 142 & 112 \\
\hline 5797 & $-142_{-14}^{+13}$ & $92_{-22}^{+22}$ & 35 & 34 \\
\hline 6196 & $-144^{f}$ & $<31$ & 12 & 12 \\
\hline 6203 & $-155_{-33}^{+19}$ & $133_{-32}^{+23}$ & 57 & 37 \\
\hline 6269 & $-120_{-14}^{+14}$ & $45_{-18}^{+18}$ & 26 & \\
\hline 6283 & $-144^{f}$ & $488_{-27}^{+90}$ & 330 & 249 \\
\hline 6613 & $-130_{-4}^{+5}$ & $137_{-14}^{+14}$ & 43 & 47 \\
\hline $\mathrm{NaD}_{1}$ & $-144_{-2}^{+2}$ & 384 & 147 & \\
\hline
\end{tabular}

${ }^{f}$ Denotes velocities held fixed during fitting.

tentatively detected. The $\lambda \lambda 4726,4501,5849,6376,6379,6445$, 6532, 6660, 6993 and 8026 DIBs were too weak to be detected. Telluric absorption line contamination prevented analysis of the $\lambda \lambda 6886,6919,7224$ and 7334 DIBs.

The radial velocities and equivalent widths of the strongest DIBs were measured using the least-squares fitting technique described by Cordiner et al. (2008). DIB profiles observed towards $\beta^{1}$ Sco (Cordiner 2006), convolved with the DEIMOS spectral point spread function, were shifted and scaled to obtain the best fit to the observed DIBs. The observed DIB spectra and fitted profiles are shown in Fig. 2.

For the $\lambda \lambda 5780,5797,6203$ and 6613 DIBs, the radial velocities and equivalent widths were allowed to vary in the fits. For the $\lambda 6196$ and $\lambda 6283$ DIBs, the radial velocities were fixed at the M33 Na I radial velocity and only the equivalent widths were allowed to vary. A telluric absorption component (shown in Fig. 2) was also included for $\lambda 6283$. For $\lambda 5705$ and $\lambda 6269$, the DIB profiles were assumed to be Gaussian. DIB radial velocities and EWs are shown in Table 2. The $\lambda 5797 E W$ is the sum of the $\lambda 5797$ and overlapping (broad) $\lambda 5795$ DIBs, which cannot be separated at the observed resolving power. The radial velocities of all measured DIBs agree closely with the Na D-line radial velocity, which is consistent with previous high resolution studies of the Galactic and extragalactic ISM (such as those by Sollerman et al. 2005 and Cox et al. 2006, 2007), and the low resolution study of M 31 by Cordiner et al. (2008). However, it should be noted that the velocity of the M33 interstellar clouds may differ from the fitted $\mathrm{Na}$ I radial velocity due to stellar contamination of the Na Dlines.

There is evidence for a Lorentzian-shaped absorption feature (with $F W H M \approx 18 \AA$ ), centered close to $4426 \AA$, which we interpret as possibly being caused by the $\lambda 4428$ DIB in M 33 . However, the low $S / N$ and presence of several stellar lines in this region prevents the precise measurement of this feature. The upper limit on the M $33 \lambda 4428 E W$ is $1.55 \AA$. 

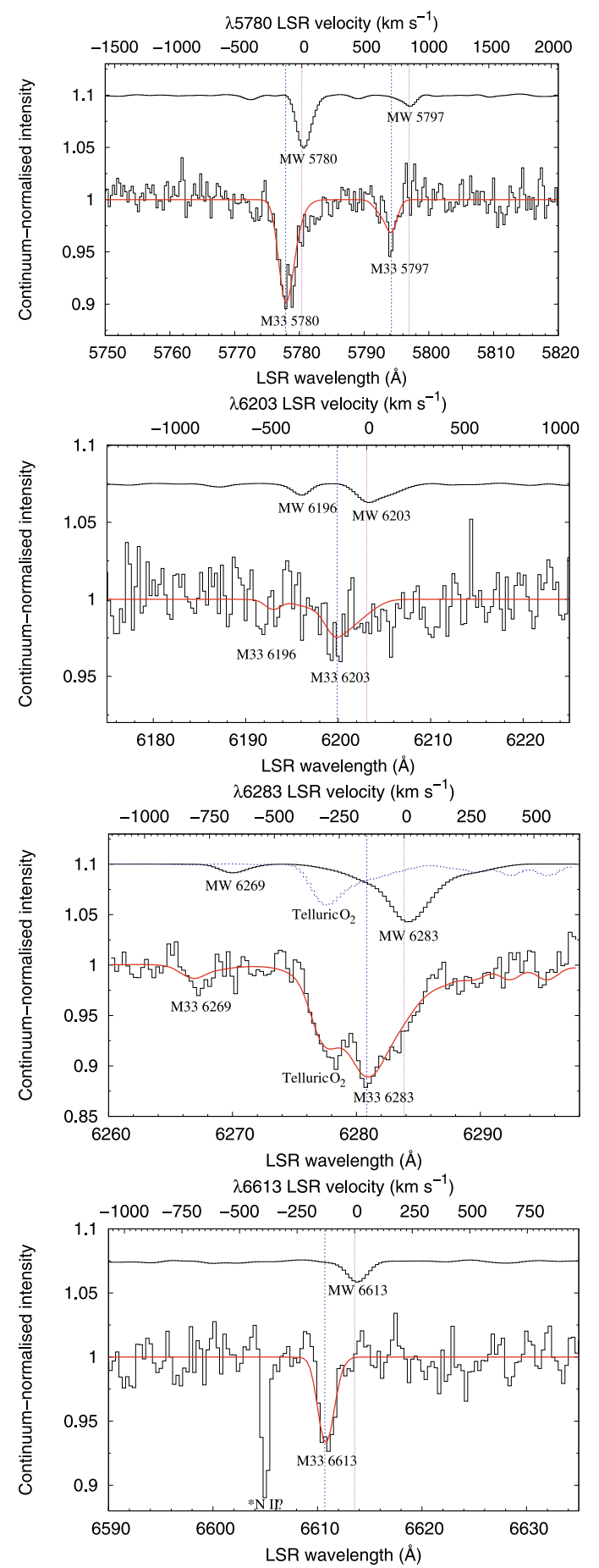

Fig. 2. Continuum-normalised spectra of the observed DIBs (lower black histograms). Fitted model DIB profiles are overlaid (thick red curves). Velocity scales at the top are relative to the Galactic DIB rest wavelengths published by Galazutdinov et al. (2000). The DIB rest wavelengths and blue-shifted M 33 DIB wavelengths are plotted with vertical dotted and dashed lines. Spectra of $\beta^{1}$ Sco are plotted as a reference for Galactic DIB wavelengths and profiles, shifted vertically for display purposes (upper black histograms). A telluric $\mathrm{O}_{2}$ spectrum is shown for the $6283 \AA$ region (dotted blue line).

Statistical equivalent width and DIB velocity error estimates were derived using the same method as Cordiner et al. (2008). To account for errors introduced into the fits due to the blending of MW and M33 DIB components for $\lambda \lambda 4428,5780$ and 6283 DIBs, a Milky Way component was also included. The
$E W \mathrm{~s}$ of these MW components were varied between zero and the value that corresponded to the least-squares fit. The resulting M 33 DIB parameter ranges are included in the errors quoted in Table 2.

\subsection{Foreground gas and dust}

LAB H I data (Kalberla et al. 2005) for the closest survey point in the direction of the target imply that $N(\mathrm{HI})=4.3 \times 10^{20} \mathrm{~cm}^{-2}$ for the velocity range of the Galactic foreground gas (from -60 to $50 \mathrm{~km} \mathrm{~s}^{-1}$ ). Equation (7) of Burstein \& Heiles (1978) is used to calculate the foreground reddening for lines of sight at latitudes away from the Galactic plane, which yields $E_{B-V}=0.03$ mag towards our targets. The foreground reddening derived by Schlegel et al. (1998) from COBE and IRAS maps is 0.042 mag.

We measure a Galactic foreground Na I column density towards J013346.96 +303642.8 of $2.8 \times 10^{12} \mathrm{~cm}^{-2}$, which corresponds to $E_{B-V} \sim 0.10 \mathrm{mag}$ (Hobbs 1974). However, there is considerable scatter in the relationship derived by Hobbs (1974) and there is likely to be contamination of the Na I profile close to $v=0$ due to sky-line subtraction residuals. We therefore adopt a foreground reddening of $0.04 \pm 0.02 \mathrm{mag}$, which results in a foreground-corrected reddening of $E_{B-V}^{\mathrm{M} 33}=0.22 \pm 0.04$ mag.

\section{Discussion}

DIB equivalent widths in the Milky Way correlate with $E_{B-V}$, and many DIBs have $E W \mathrm{~s}$ that correlate with each other (Herbig 1995). In terms of the relative strengths of the detected DIBs and their (Doppler-shifted) wavelengths, the observed M 33 DIB spectrum is consistent with that observed in the Galaxy and in M31 (by comparison with Cordiner et al. 2008). Only the strongest known Galactic DIBs were detected in M33, which is as expected given the signal-to-noise and the low reddening of the target.

The observed DIBs are stronger by a factor of about two compared with those observed towards our chosen Galactic reference target $\beta^{1}$ Sco. The $\beta^{1}$ Sco sightline has $E_{B-V}=0.22$, which is identical to the $E_{B-V}^{\mathrm{M} 33}$ value for J013346.96+303642.8. As shown in Table 2, the $\beta^{1}$ Sco DIBs are about as strong as the Galactic average per unit $E_{B-V}$. The MW Avg.@ $E_{B-V}^{\mathrm{M} 33}$ values in Table 2 were calcualted by taking the average of $E W / E_{B-V}$ for each DIB in the data published by Herbig (1993), Thorburn et al. (2003), Megier et al. (2005), and Cordiner (2006), then scaling by a factor of $E_{B-V}^{\mathrm{M} 33}=0.22$. Figure 3 shows that the M 33 $\lambda \lambda 5780,6283$ and $6613 \mathrm{EW} / E_{B-V}$ values are similar to the upper limit to the spread of values observed in the MW, and are almost as large as observed towards MAG 70817 in M 31 (the upper left (blue) square in the plots). In the Milky Way, such strong DIBs (per unit reddening), are rarely, if ever, observed. The Galactic target HD 164402 has a $\lambda 5780 E W$ among the largest known for its reddening $\left(E_{B-V}=0.22, E W(5780)=187 \mathrm{~m} \AA\right)($ Herbig 1993), which is still significantly lower than found here. Such large DIB strengths are remarkable, but cannot be considered as indicative of the properties of DIBs throughout M33 because this target was selected from the observed set due to its strong DIBs. In the direction of the observed target, the $21 \mathrm{~cm} \mathrm{H} \mathrm{I} \mathrm{emis-}$ sion maximum is at about $-150 \mathrm{~km} \mathrm{~s}^{-1}$ (Warner et al. 1973), which is consistent with the measured $\mathrm{Na}$ I and DIB velocities.

$\mathrm{J} 013346.96+303642.8$ is $3.0^{\prime}(730 \mathrm{pc})$ from the centre of M33 (assuming a distance of $840 \mathrm{kpc}$ ). Observations of $\mathrm{H} \mathrm{II}$ regions in M 33 by Magrini et al. (2007) indicate that the metallicity at this radius is approximately solar. This suggests 


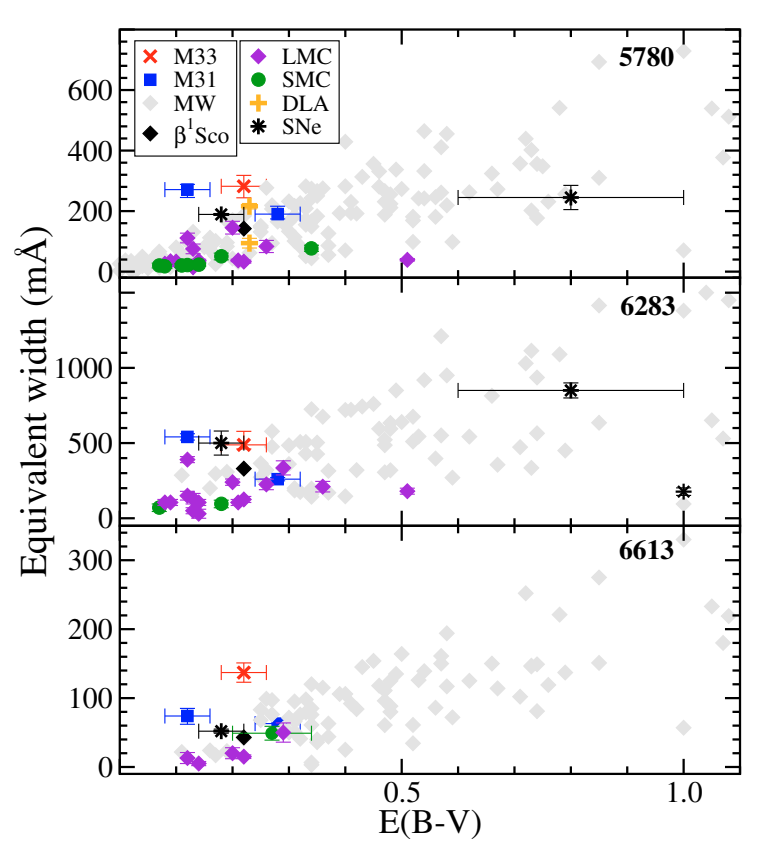

Fig. 3. $\lambda \lambda 5780,6283$ and 6613 equivalent widths vs. reddening for different galaxies. Milky Way data (from Herbig 1993; Thorburn et al. 2003; Megier et al. 2005; Cordiner 2006), are shown as grey diamonds. Data are shown for M 33 (this work), M 31 (Cordiner et al. 2008), the LMC and SMC (Welty et al. 2006), DLAs (York et al. 2006; Ellison et al. 2008) and supernovae in NGC 1448 and NGC 2770 (Sollerman et al. 2005; Thoene et al. 2008).

that the metallicity of the M 33 ISM along the line of sight to this star should be comparable with that in the solar neighbourhood. Thus, an explanation other than metallicity may be required to explain the large DIB strengths.

The GALEX map of M33 (Thilker et al. 2005) shows that the observed star is close to (within $0.4^{\prime} / 96 \mathrm{pc}$ ) a small cluster of UV-bright stars. It has been suggested that UV radiation is required for the production of DIB carriers (see Herbig 1995; Kendall et al. 2002), which may explain why the DIBs along this sightline are strong. This hypothesis could be tested by observing DIBs along sightlines that traverse UV-bright sources with varying impact parameters. Figure 2 shows that the observed ratio of $\lambda 5780$ to $\lambda 5797$ DIB strengths is similar to that of $\beta^{1}$ Sco and that this M 33 sightline is therefore of " $\sigma$ " type (Weselak et al. 2000). This designation may be considered as evidence that the interstellar gas is relatively diffuse and quite strongly UV-irradiated (see e.g. Cami et al. 1997), although not to such a degree as to cause nebular emission, which tends to be associated with weaker DIBs (Snow et al. 1995). High resolution spectroscopic observations of interstellar Ca I, Ca II, CH, and CN near-UV absorption lines would provide a further probe of the UV field strength along this sightline; in this respect, the upper limits to the column densities of these species derived from our spectra are inconclusive.

\section{Conclusion}

The $\lambda \lambda 5705,5780,5797,6203,6269,6283$, and 6613 DIBs have been detected in M33 towards the A3 Ia star $\mathrm{J} 013346.96+303642.8$ at velocities corresponding (within $25 \mathrm{~km} \mathrm{~s}^{-1}$ ) to the mean M33 Na I absorption velocity. This constitutes the first reported detection of DIBs in the M33 galaxy. Both the $\lambda 4428$ and $\lambda 6196$ DIBs are tentatively detected. The overall spectrum of DIBs observed in M33, including the strength ratios between different DIBs, is found to be similar to that observed in the Milky Way. However, in this line-of-sight, the DIBs measured are up to about a factor of two stronger per unit reddening than towards the Galactic reference target $\beta^{1}$ Sco (which has the same reddening, $E_{B-V}=0.22$ ). The $\lambda \lambda 5780$, 6283 and 6613 equivalent widths per unit $E_{B-V}$ are among the highest observed in any galaxy. The high DIB strengths may be due to differences between the gas/dust composition or interstellar radiation field of M 33 and the Galaxy. Further observational and theoretical studies of the chemical and physical properties of the ISM along this sightline will be required to determine what may be causing such strong DIBs.

Acknowledgements. The W. M. Keck Observatory is operated as a scientific partnership among the California Institute of Technology, the University of California, and NASA. The observatory was made possible by the generous financial support of the W. M. Keck Foundation. The analysis pipeline used to reduce the Keck/DEIMOS data was developed at UC Berkeley with support from NSF grant AST-0071048. M.A.C. thanks QUB for financial support. K.T.S. thanks EPSRC for a studentship. N.L.J.C. acknowledges support from the Faculty of the European Space Astronomy Centre (ESAC).

\section{References}

Burstein, D., \& Heiles, C. 1978, ApJ, 225, 40

Cami, J., Sonnentrucker, P., Ehrenfreund, P., \& Foing, B. H. 1997, A\&A, 326, 822

Cordiner, M. A. 2006, Ph.D. Thesis, The University of Nottingham Cordiner, M. A., Cox, N. L. J., Trundle, C., et al. 2008, A\&A, 480, L13 Cox, N. L. J., \& Patat, F. 2008, A\&A, 485, L9

Cox, N. L. J., Cordiner, M. A., Cami, J., et al. 2006, A\&A, 447, 991

Cox, N. L. J., Cordiner, M. A., Ehrenfreund, P., et al. 2007, A\&A, 470, 941

Ehrenfreund, P., \& Foing, B. H. 1996, A\&A, 307, L25

Ehrenfreund, P., Cami, J., Jiménez-Vicente, J., et al. 2002, ApJ, 576, L117

Ellison, S. L., York, B. A., Murphy, M. T., et al. 2008, MNRAS, 383, L30

Evans, C. J., \& Howarth, I. D. 2003, MNRAS, 345, 1223

Evans, C. J., Howarth, I. D., Irwin, M. J., Burnley, A. W., \& Harries, T. J. 2004, MNRAS, 353, 601

Galazutdinov, G. A., Musaev, F. A., Krełowski, J., \& Walker, G. A. H. 2000, PASP, 112,648

Herbig, G. H. 1993, ApJ, 407, 142

Herbig, G. H. 1995, ARA\&A, 33, 19

Hobbs, L. M. 1974, ApJ, 191, 381

Howarth, I. D., Price, R. J., Crawford, I. A., \& Hawkins, I. 2002, MNRAS, 335, 267

Jenniskens, P., \& Desert, F.-X. 1994, A\&AS, 106, 39

Kalberla, P. M. W., Burton, W. B., Hartmann, D., et al. 2005, A\&A, 440, 775

Kendall, T. R., Mauron, N., McCombie, J., \& Sarre, P. J. 2002, A\&A, 387, 624

Kurucz, R. 1993, ATLAS9 Stellar Atmosphere Programs and $2 \mathrm{~km} \mathrm{~s}^{-1}$ Grid,

Kurucz CD-ROM No. 13 (Cambridge, MA: Smithsonian Astrophysical Observatory)

Magrini, L., Vílchez, J. M., Mampaso, A., Corradi, R. L. M., \& Leisy, P. 2007, A\&A, 470, 865

Massey, P., Olsen, K. A. G., Hodge, P. W., et al. 2006, AJ, 131, 2478

Megier, A., Krełowski, J., \& Weselak, T. 2005, MNRAS, 358, 563

Sarre, P. J. 2006, J. Mol. Spectr., 238, 1

Sarre, P. J., Miles, J. R., Kerr, T. H., et al. 1995, MNRAS, 277, L41

Schlegel, D. J., Finkbeiner, D. P., \& Davis, M. 1998, ApJ, 500, 525

Snow, T. P. 2002, in 17th IAP Colloq., on Gaseous Matter in Galaxies and Intergalactic Space, ed. R. Ferlet, M. Lemoine, J. M. Désert, \& B. Raban, 63

Snow, T. P., Bakes, E. L. O., Buss, Jr., R. H., \& Seab, C. G. 1995, A\&A, 296, L37

Sollerman, J., Cox, N., Mattila, S., et al. 2005, A\&A, 429, 559

Thilker, D. A., Hoopes, C. G., Bianchi, L., et al. 2005, ApJ, 619, L67

Thoene, C. C., Michalowski, M. J., Leloudas, G., et al. 2008, in preparation

Thorburn, J. A., Hobbs, L. M., McCall, B. J., et al. 2003, ApJ, 584, 339

Warner, P. J., Wright, M. C. H., \& Baldwin, J. E. 1973, MNRAS, 163, 163

Welty, D. E., Federman, S. R., Gredel, R., Thorburn, J. A., \& Lambert, D. L. 2006, ApJS, 165, 138

Weselak, T., Schmidt, M., \& Krełowski, J. 2000, A\&AS, 142, 239

York, B. A., Ellison, S. L., Lawton, B., et al. 2006, ApJ, 647, L29 